\title{
A Chronic, Massive Thrombus in the Right Main Pulmonary Artery
} A Case Report and Echocardiographic Analysis

\author{
Ichiro Murata, MD; Makoto Sonoda, MD; Fumiyoshi Watanabe, MD; \\ Weidong Yang, MD; Katsu Takenaka, MD*
}

\begin{abstract}
A chronic, large thrombus in the right main pulmonary artery (PA) was detected in a 54-year-old woman with a history of surgical repair of atrial septal defect. Color flow imaging revealed a prominent red signal along the right border of the markedly dilated PA. Pulsed Doppler echocardiography showed that the red signal was caused by flow reversal occurring during systole. According to the physics of blood flow, flow reversal probably represents secondary, helical flow, which may be related to thrombus formation. (Jpn Circ J 1999; 63: 312-314)
\end{abstract}

Key Words: Blood flow; Echocardiography; Pulmonary artery (PA); Thrombus

$\mathbf{T}$ hromboembolism of the main pulmonary arteries (pulmonary trunk and its proximal branches) may be acute, causing cardiopulmonary shock, or it may manifest itself as chronic pulmonary hypertension! There have been numerous case reports on this disorder? ${ }^{2-7}$ Although most cases of pulmonary artery (PA) thrombosis are embolic in nature, in situ thrombosis has also been suggested8 Because pulmonary thrombi are expected to dissolve completely by intrinsic fibrinolysis, the pathogenesis of chronic, large thrombi in the main PAs is unclear. Here, we report a case of a chronic, massive thrombus in the right main PA in a patient who had undergone surgical repair of an atrial septal defect. We also examined blood flow characteristics in the main PA with Doppler echocardiography to obtain an insight into the pathogenesis of the thrombus.

\section{Case Report}

A 54-year-old woman, complaining of exertional dyspnea, was seen in July 1996. She had undergone surgical repair of an ostium secundum atrial septal defect at the age of 38 and total hysterectomy for myoma at the age of 46. She was a short, well-nourished woman in no respiratory distress at rest, with a blood pressure of $120 / 84 \mathrm{mmHg}$ and a heart rate of $84 / \mathrm{min}$. Cardiac auscultation revealed an accentuated pulmonary component of the second heart sound, a grade $2 / 6$ systolic murmur at the second intercostal space, and a grade $2 / 6$ holosystolic murmur at the fifth intercostal space over the left parasternal border. Chest radiography revaled cardiomegaly and markedly dilated PAs. ECG revealed right ventricular hypertrophy.

The blood cell count revealed a hematocrit of $41 \%$, a

(Received October 21, 1998; revised manuscript received January 8, 1999; accepted January 11, 1999)

Department of Cardiovascular Medicine, Graduate School of Medicine, and *Department of Laboratory Medicine, University of Tokyo, Tokyo, Japan

Mailing address: Ichiro Murata, MD, The Department of Cardiovascular Medicine, Graduate School of Medicine, University of Tokyo, 7-3-1 Hongo, Bunkyo-ku, Tokyo 113-8655, Japan white cell count of $3400 / \mathrm{mm}^{3}$ and a platelet count of $10.5 \times 10^{4} / \mathrm{mm}^{3}$. The plasma prothrombin activity was $68 \%$. Values of protein S, protein C, antithrombin III, and anticardiolipin antibody were within the normal limits.

Multiplane transesophageal echocardiography (TEE) revealed markedly dilated right and left PAs ( $46 \mathrm{~mm}$ and 48 $\mathrm{mm}$ at maximum diameter) and a non-occlusive thrombus $(3 \times 7 \mathrm{~cm})$ with a homogeneous ultrasonic characteristic attached to the posterior wall of the right PA (Fig 1). A computerized tomographic (CT) scan revealed a large thrombus in the right PA and no thrombus in the left PA. A pulmonary perfusion scan showed no significant defects. A right heart catheterization showed a mean PA pressure of $33 \mathrm{mmHg}$, a mean pulmonary capillary wedge pressure of $11 \mathrm{mmHg}$, a mean right atrial pressure of $10 \mathrm{mmHg}$, and a pulmonary vascular resistance of 433 dyne $\mathrm{s}^{-1} \mathrm{~cm}^{5}$. The systolic pressure gradient was not noted between the right ventricle and the PA. Pulmonary angiography showed markedly dilated PAs with no significant flow obstruction.

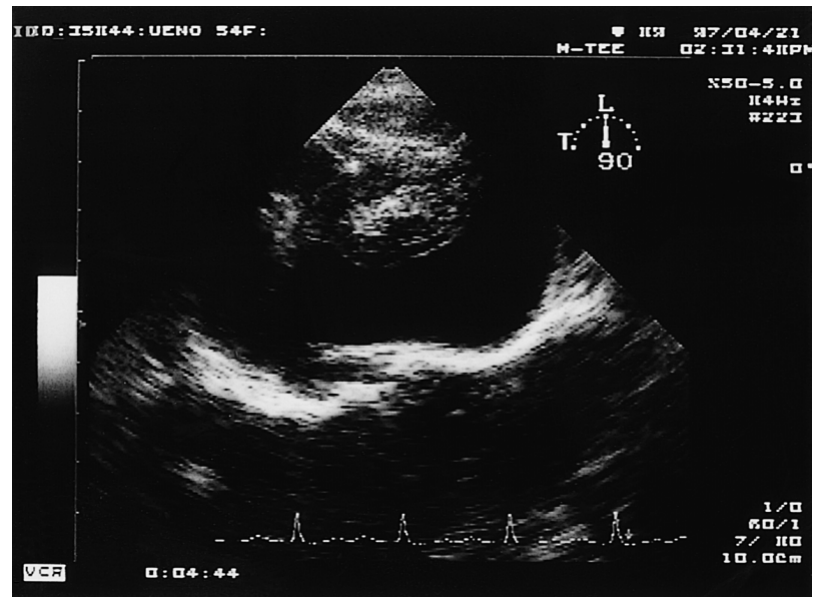

Fig 1. Multiplane TEE shows a large non-occlusive thrombus with a homogeneous ultrasonic characteristic in the right pulmonary artery (at 90 degrees). The thrombus is immobile and adherent to the posterior wall with a broad basilar attachment. 
Venous Doppler studies of the lower extremities were negative.

Transthoracic color flow imaging using a commercially available system (Aloka SSD-2200) revealed a prominent red signal along the right border of the dilated main PA (Fig 2, left). Pulsed Doppler examination with a sample volume in the red signal demonstrated that it was caused by flow reversal, which occurred immediately after the systolic flow, with its velocity peaking in late systole, and persisted throughout diastole (Fig 2, right). Decreased forward velocity and persistent flow reversal were observed in the proximal right PA. Just proximal to the thrombus, marked reductions in forward and reverse flow velocities suggested flow stasis (Fig 3). In contrast, the flow at the proximal left PA was predominantly forward with a peak velocity of $36 \mathrm{~cm} / \mathrm{s}$ (Fig 4$)$.

Warfarin, ticlopidine and diltiazem were prescribed, with a gradual improvement in the symptoms. However,

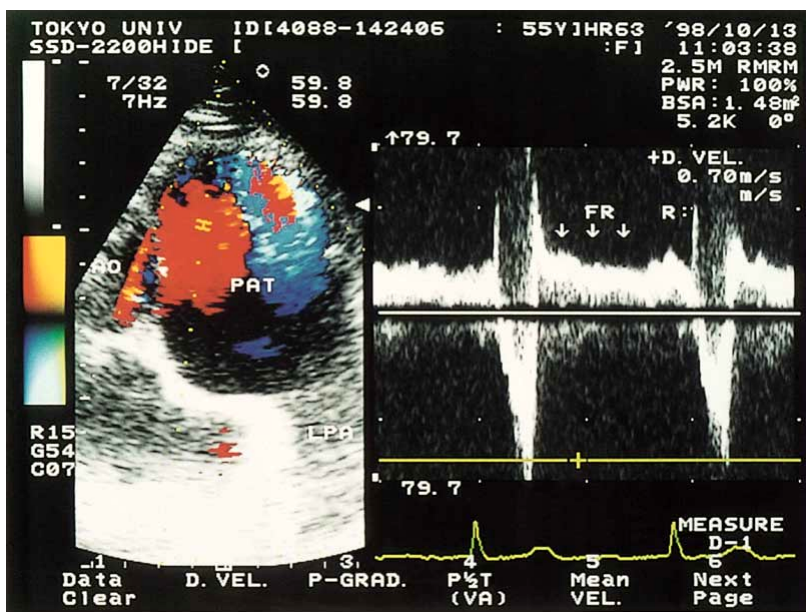

Fig 2. Transthoracic color flow imaging revealed a prominent red signal along the right border of the dilated main PA (left). Simultaneous pulsed Doppler recording with a sample volume in the red signal demonstrated flow reversal (arrows), which occurred immediately after the systolic flow (peak velocity, $70 \mathrm{~cm} / \mathrm{s}$ ), with its velocity peaking in late systole, and persisted throughout diastole (right). PAT, pulmonary trunk; AO, aortic orifice; LPA, left pulmonary artery. repeat TEE studies performed 6 and 12 months later revealed no significant change in the size of the thrombus.

\section{Discussion}

Echocardiographic and CT data in this case suggest a chronic PA thrombus that has become resistant to anticoagulation. Chronic left-to-right shunting most likely caused the marked dilatation of the PAs, which persisted after the surgical repair of the atrial septal defect. It is unclear whether the thrombus formed in situ or had embolized from leg veins. In either case, blood flow stasis in the markedly dilated PAs had probably contributed to PA thrombosis. However, persistence of a large mural thrombus in the right PA suggests the presence of a localized factor. In this respect, it is noteworthy that the Doppler echocardiographic examination demonstrated prominent flow reversal along the right PA border.

When echocardiographically visualized in a left parasternal short-axis view, the right ventricular outflow tract and the main PA form a curved tube on the left side of the aorta. According to the physics of blood flow in a curved tube, it would be expected that the primary axial velocities of systolic flow would be directed toward the left PA border. Slower moving blood cells located in the main PA during diastole would be forced back toward the right border, resulting in a helical motion of systolic flow, ie, the socalled secondary flow? Therefore, the flow reversal observed in our patient may represent this secondary flow, which probably caused a reduction in forward flow velocity in the right PA. Sung and Yoganathan, using an in vitro PA model, observed that secondary flow was much stronger in the right PA than in the left PA, and attributed this to the different bifurcation angles of the right and left PAs $!^{10}$ If their finding can be extrapolated to physiological hemodynamics, prominent secondary flow could disrupt the endothelial integrity and contribute to in situ thrombogenesis. The exact cause of thrombus formation in this case remains unclear. However, we speculate that both secondary flow and flow stasis have been thrombogenic in this case.

Most case reports on main PA thrombi have focused on the diagnosis ${ }^{2-4}$ and treatment ${ }^{5-7}$ of this condition. Rittoo et al reported the case of a patient with a thrombus in the right
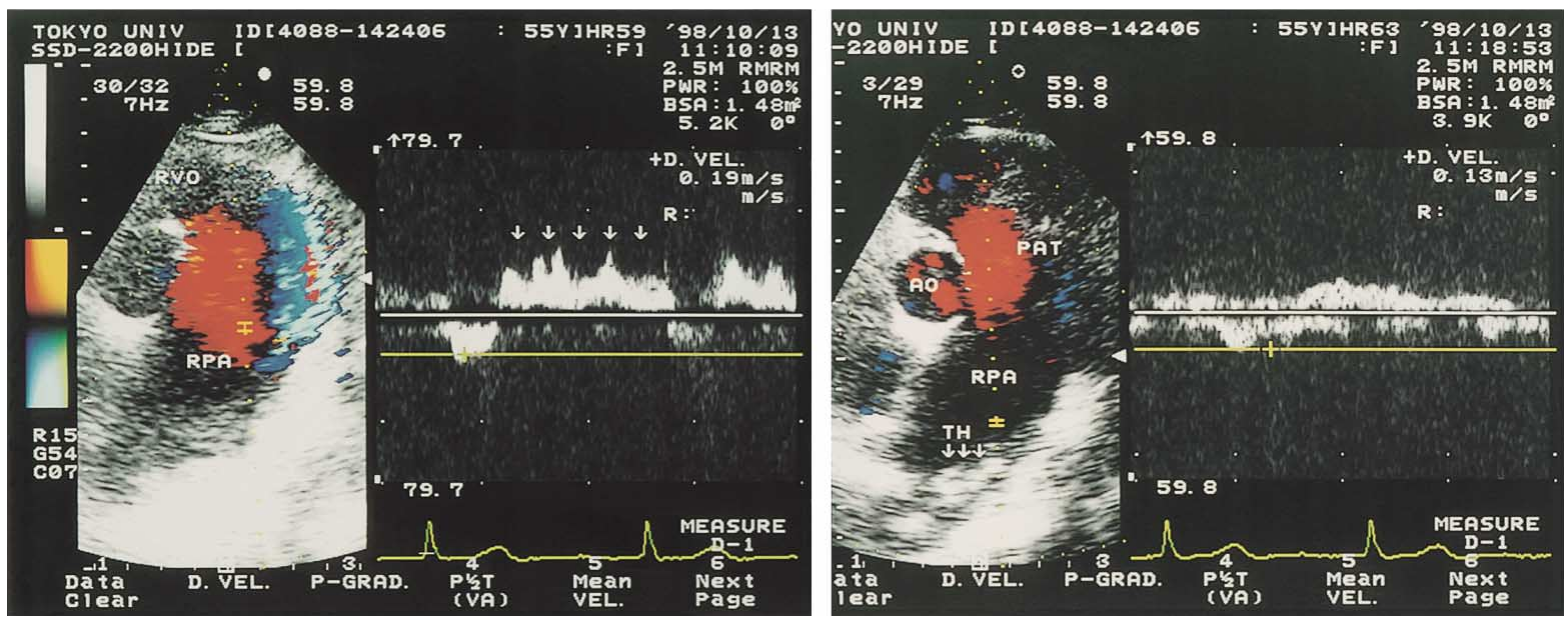

Fig 3. At the proximal right PA, persistent flow reversal (arrows) and decreased velocity of forward flow $(19 \mathrm{~cm} / \mathrm{s})$ were observed. Marked reductions in forward and reversed flow velocities suggested flow stasis just proximal to the thrombus. RVO, right ventricular outflow tract; RPA, right pulmonary artery; AO, aortic orifice; PAT, pulmonary trunk; TH, thrombus. 


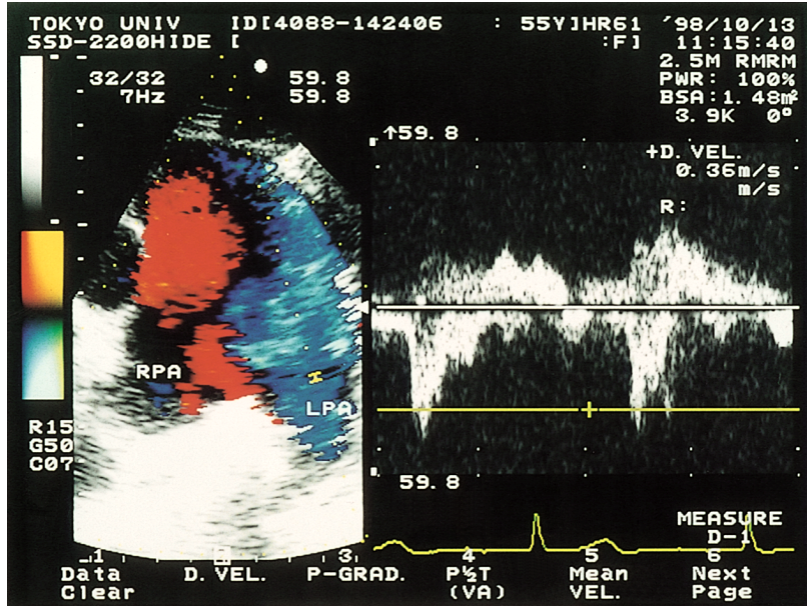

Fig 4. At the proximal left PA, a forward flow is evident, with a peak velocity of $36 \mathrm{~cm} / \mathrm{s}$. The pulsed Doppler recording also shows flow reversal.

PA after surgical closure of an atrial septal defect, as in our case? However, to our knowledge, the relationship between thrombus formation and flow characteristics has not been previously studied. Wittlich et al performed transesophageal echocardiography in 60 patients with severe pulmonary embolism and pulmonary hypertension? They detected main PA thrombi much more frequently in the right PA than in the left (26 compared with 3 ), which could be partly attributable to relatively poor visualization of the left PA by transesophageal approach. However, the findings in our case suggest that secondary flow may predispose a floating thrombus to embolize in the right PA.

\section{References}

1. Rich S, Levitsky S, Brundage BH: Pulmonary hypertension from chronic pulmonary thromboembolism. Ann Intern Med 1988; 108: $425-434$

2. Wittlich N, Erbel R, Eichler A, Schuster S, Jakob H, Iversen S, et al: Detection of central pulmonary artery thromboemboli by transesophageal echocardiography in patients with severe pulmonary embolism. J Am Soc Echocardiogr 1992; 5: 515-524

3. Rittoo D, Sutherland GR, Samuel L, Flapan AD, Shaw TRD: Role of transesophageal echocardiography in diagnosis and management of central pulmonary artery thromboembolism. Am J Cardiol 1993; 71: $1115-1118$

4. Ohkura H, Yoshikawa J, Yoshida K, Akasaka T, Shakudo M, Takagi $\mathrm{T}$, et al: Value and limitations of transesophageal echocardiographic detection of pulmonary thrombi in the diagnosis of pulmonary embolism (in Japanese with English abstract). Jpn J Med Ultrasonics 1994; 21: $93-97$

5. Moser KM, Daily PO, Peterson K, Dembitsky W, Vapnek JM, Schure D, et al: Thromboendarterectomy for chronic, major-vessel thromboembolic pulmonary hypertension. Immediate and long-term results in 42 patients. Ann Intern Med 1987; 107: 560-565

6. Nakajima T, Ando H, Ueno Y, Umadome H, Takahashi Y, Okano Y, et al: Successful thromboendarterectomy for chronic pulmonary embolism in a patient with systemic lupus erythematosus and antiphospholipid syndrome. Jpn Circ J 1997; 61: 958-964

7. Mihara H, Araki M, Yasuda T, Handa K, Tanaka K: Use of temporary vena cava filters after catheter-directed fragmentation and thrombolysis in patients with acute pulmonary thromboembolism. Jpn Circ J 1998; 62: 462-464

8. Moser KM, Fedullo PF, Finkbeiner WE, Golden J: Do patients with primary pulmonary hypertension develop extensive central thrombi? Circulation 1995; 91: 741-745

9. Cape EG, Sung HW, Yoganathan AP: Physics of blood flow. In Nanda NC, editor. Doppler Echocardiography, 2nd edn. Philadelphia: Lea \& Febiger, 1993: 24-34

10. Sung HW, Yoganathan AP: Secondary flow velocity patterns in a pulmonary artery model with varying degrees of valvular pulmonic stenosis: pulsatile in vitro studies. J Biomech Eng 1990; 112: 88-92 